\title{
Hard-photon production and tests of QED at LEP
}

\section{L3 Collaboration}

M. Acciarri ${ }^{\mathrm{z}}$, P. Achard s , O. Adriani ${ }^{\mathrm{p}}$, M. Aguilar-Benitez ${ }^{\mathrm{y}}$, J. Alcaraz ${ }^{\mathrm{y}}$, G. Alemanni v , J. Allaby ${ }^{\mathrm{q}}$, A. Aloisio ${ }^{\mathrm{ab}}$, M.G. Alviggi ab , G. Ambrosi ${ }^{\mathrm{s}}$, H. Anderhub au, V.P. Andreev f,aj, T. Angelescu ${ }^{1}$, F. Anselmo i, A. Arefiev ${ }^{\text {aa }}$, T. Azemoon ${ }^{\text {c }}$, T. Aziz ${ }^{\text {j }, ~ P . ~ B a g n a i a ~ a i ~, ~ L . ~ B a k s a y ~}{ }^{\text {ap }}$, A. Balandras ${ }^{\text {d }}$, R.C. Ball ${ }^{\text {c }}$, S. Banerjee ${ }^{j}$, Sw. Banerjee ${ }^{j}$, A. Barczyk ${ }^{\text {au,as }}$, R. Barillère ${ }^{q}$, L. Barone ${ }^{\text {ai }}$, P. Bartalini ${ }^{\mathrm{v}}$, M. Basile ${ }^{\mathrm{i}}$, R. Battiston ${ }^{\text {af }}$, A. Bay ${ }^{\mathrm{v}}$, F. Becattini ${ }^{\mathrm{p}}$, U. Becker ${ }^{\mathrm{n}}$, F. Behner ${ }^{\mathrm{au}}$, L. Bellucci ${ }^{\mathrm{p}}$, J. Berdugo ${ }^{\mathrm{y}}$, P. Berges ${ }^{\mathrm{n}}$, B. Bertucci af , B.L. Betev ${ }^{\text {au }}$, S. Bhattacharya ${ }^{j}$, M. Biasini af ${ }^{\text {af A. Biland }}{ }^{\text {au }}$, J.J. Blaising ${ }^{d}$, S.C. Blyth ${ }^{\text {ag }}$, G.J. Bobbink ${ }^{\text {b }}$, A. Böhm ${ }^{\text {a }}$, L. Boldizsar ${ }^{\mathrm{m}}$, B. Borgia ${ }^{\text {ai }}$, D. Bourilkov ${ }^{\text {au }}$,


A. Buffini ${ }^{\text {p }}$, A. Buijs ${ }^{\text {aq }}$, J.D. Burger ${ }^{\text {n }}$, W.J. Burger ${ }^{\text {af }}$, A. Button ${ }^{\text {c }}$, X.D. Cai ${ }^{\text {n, }}$, M. Campanelli au, M. Capell ${ }^{\mathrm{n}}$, G. Cara Romeo ${ }^{\mathrm{i}}$, G. Carlino ${ }^{\mathrm{ab}}$, A.M. Cartacci ${ }^{\mathrm{p}}$, J. Casaus ${ }^{\mathrm{y}}$, G. Castellini ${ }^{\mathrm{p}}$, F. Cavallari ${ }^{\text {ai }}$, N. Cavallo ${ }^{\mathrm{ab}}$, C. Cecchi ${ }^{\text {af }}$, M. Cerrada ${ }^{\mathrm{y}}$, F. Cesaroni ${ }^{\text {w }}$, M. Chamizo ${ }^{\text {s, }, ~ Y . H . ~ C h a n g ~}{ }^{\text {aw }}$, U.K. Chaturvedi ${ }^{\mathrm{r}}$, M. Chemarin ${ }^{\mathrm{x}}$, A. Chen ${ }^{\text {aw }}$, G. Chen ${ }^{\mathrm{g}}$, G.M. Chen ${ }^{\mathrm{g}}$, H.F. Chen ${ }^{\mathrm{t}}$, H.S. Chen ${ }^{\mathrm{g}}$, G. Chiefari ${ }^{\mathrm{ab}}$, L. Cifarelli ak, F. Cindolo ${ }^{\text {i }}$, C. Civinini ${ }^{\text {p }}$, I. Clare ${ }^{\text {n }}$, R. Clare ${ }^{\text {n }}$, G. Coignet ${ }^{\text {d }}$, A.P. Colijn ${ }^{\mathrm{b}}$, N. Colino ${ }^{\mathrm{y}}$, S. Costantini ${ }^{\mathrm{e}}$, F. Cotorobai ${ }^{\mathrm{l}}$, B. Cozzoni ${ }^{\mathrm{i}}$, B. de la Cruz ${ }^{\mathrm{y}}$, A. Csilling ${ }^{\mathrm{m}}$, S. Cucciarelli af ${ }^{\text {, T.S. Dai }}{ }^{\mathrm{n}}$, J.A. van Dalen ${ }^{\text {ad }}$, R. D’Alessandro ${ }^{\mathrm{p}}$, R. de Asmundis ${ }^{\mathrm{ab}}$, P. Déglon ${ }^{\mathrm{s}}$, A. Degré ${ }^{\mathrm{d}}, \mathrm{K}$. Deiters ${ }^{\text {as }}$, D. della Volpe ${ }^{\text {ab }}$, P. Denes ${ }^{\text {ah }}$, F. DeNotaristefani ${ }^{\text {ai }}$, A. De Salvo ${ }^{\text {au }}$, M. Diemoz ${ }^{\text {ai }}$, D. van Dierendonck ${ }^{\mathrm{b}}$, F. Di Lodovico ${ }^{\text {au }}$, C. Dionisi ${ }^{\text {ai }}$, M. Dittmar ${ }^{\text {au }}$, A. Dominguez ${ }^{\text {al }}$, A. Doria ${ }^{\text {ab }}$, M.T. Dova ${ }^{\text {r,1 }}$, D. Duchesneau ${ }^{\text {d, }}$ D. Dufournaud ' , P. Duinker b, I. Duran am , H. El Mamouni ${ }^{x}$, A. Engler ${ }^{\mathrm{ag}}$, F.J. Eppling ${ }^{n}$, F.C. Erné ${ }^{\text {b }}$, P. Extermann ${ }^{\text {s }}$, M. Fabre ${ }^{\text {as }}$, R. Faccini ${ }^{\text {ai }}$, M.A. Falagan ${ }^{y}$, S. Falciano ${ }^{\text {ai, }}$, A. Favara ${ }^{\mathrm{q}}$, J. Fay ${ }^{\mathrm{x}}$, O. Fedin ${ }^{\text {aj }}$, M. Felcini ${ }^{\text {au }}$, T. Ferguson ${ }^{\text {ag }}$, F. Ferroni ai, H. Fesefeldt ${ }^{\text {a }}$, E. Fiandrini ${ }^{\text {af }}$, J.H. Field ${ }^{\text {s }}$, F. Filthaut ${ }^{\mathrm{q}}$, P.H. Fisher ${ }^{n}$, I. Fisk ${ }^{\text {al }}$, G. Forconi ${ }^{n}$, L. Fredj ${ }^{\text {s }}$, K. Freudenreich ${ }^{\text {au }}$, C. Furetta ${ }^{\mathrm{z}}$, Yu. Galaktionov aa,n, S.N. Ganguli ${ }^{j}$, P. Garcia-Abia ${ }^{\mathrm{e}}$, M. Gataullin ${ }^{\text {ae }}$, S.S. Gau ${ }^{\mathrm{k}}$, 
S. Gentile ${ }^{\mathrm{ai}, \mathrm{q}}$, N. Gheordanescu ', S. Giagu ${ }^{\text {ai }}$, Z.F. Gong ${ }^{\mathrm{t}}$, G. Grenier ${ }^{\mathrm{x}}$, O. Grimm ${ }^{\text {au }}$, M.W. Gruenewald ${ }^{\text {h }}$, M. Guida ${ }^{\text {ak }}$, R. van Gulik ${ }^{\text {b }, ~ V . K . ~ G u p t a ~}{ }^{\text {ah }}$, A. Gurtu ${ }^{\text {j, L.J. Gutay }}{ }^{\text {ar }}$, D. Haas ${ }^{\text {e }}$, A. Hasan ${ }^{\text {ac }}$, D. Hatzifotiadou ${ }^{\text {i }}$, T. Hebbeker ${ }^{\text {h }}$, A. Hervé ${ }^{\mathrm{q}}$, P. Hidas ${ }^{\mathrm{m}}$, J. Hirschfelder ${ }^{\mathrm{ag}}$, H. Hofer ${ }^{\mathrm{au}}$, G. Holzner ${ }^{\text {au }}$, H. Hoorani ${ }^{\mathrm{ag}}$, S.R. Hou ${ }^{\text {aw }}$, I. Iashvili at, B.N. Jin ${ }^{\mathrm{g}}$, L.W. Jones ${ }^{\mathrm{c}}$, P. de Jong ${ }^{\mathrm{b}}$, I. Josa-Mutuberría ${ }^{\mathrm{y}}$, R.A. Khan ${ }^{\text {r }}$, M. Kaur ${ }^{\text {r,2 }}$, M.N. Kienzle-Focacci ${ }^{\text {s, D. Kim }}{ }^{\text {ai }}$, D.H. Kim ${ }^{\text {ao }}$, J.K. Kim ${ }^{\text {ao }}$, S.C. Kim ${ }^{\text {ao }}$, J. Kirkby ${ }^{\text {q }}$, D. Kiss ${ }^{\text {m }}$, W. Kittel ${ }^{\text {ad }}$, A. Klimentov ${ }^{\text {n,aa }}$, A.C. König ${ }^{\text {ad }}$, A. Kopp ${ }^{\text {at }}$, V. Koutsenko ${ }^{\text {n,aa }}$, M. Kräber ${ }^{\text {au }}$, R.W. Kraemer ${ }^{\text {ag }}$, W. Krenz ${ }^{\text {a }}$, A. Krüger ${ }^{\text {at }}$, A. Kunin ${ }^{\text {n,aa }}$, P. Ladron de Guevara ${ }^{y}$, I. Laktineh ${ }^{\mathrm{x}}$, G. Landi ${ }^{p}$, K. Lassila-Perini ${ }^{\text {au }}$, M. Lebeau ${ }^{\mathrm{q}}$, A. Lebedev ${ }^{\mathrm{n}}$, P. Lebrun ${ }^{\mathrm{x}}$, P. Lecomte ${ }^{\text {au }}$, P. Lecoq ${ }^{\text {q }}$, P. Le Coultre ${ }^{\text {au }}$, H.J. Lee ${ }^{\text {h }}$, J.M. Le Goff ${ }^{\text {q }}$, R. Leiste ${ }^{\text {at }}$, E. Leonardi ai ${ }^{\text {ai }}$ P. Levtchenko ${ }^{\text {aj }}$, C. Li ${ }^{\text {t }}$, S. Likhoded ${ }^{\text {at }}$, C.H. Lin ${ }^{\text {aw }}$, W.T. Lin ${ }^{\text {aw }}$, F.L. Linde ${ }^{b}$, L. Lista ${ }^{a b}$, Z.A. Liu ${ }^{g}$, W. Lohmann ${ }^{\text {at }}$, E. Longo ${ }^{\text {ai }}$, Y.S. Lu ${ }^{g}$, K. Lübelsmeyer a, C. Luci q,ai , D. Luckey ${ }^{n}$, L. Lugnier ${ }^{x}$, L. Luminari ai, W. Lustermann ${ }^{\text {au }}$, W.G. Ma ${ }^{\text {t }}$, M. Maity ${ }^{j}$, L. Malgeri ${ }^{\mathrm{q}}$, A. Malinin ${ }^{\mathrm{q}}$, C. Maña ${ }^{\mathrm{y}}$, D. Mangeol ${ }^{\text {ad }}$, P. Marchesini au, G. Marian ${ }^{\circ}$, J.P. Martin ${ }^{\mathrm{x}}$, F. Marzano ${ }^{\text {ai }}$, G.G.G. Massaro b , K. Mazumdar ${ }^{\mathrm{j}}$, R.R. McNeil ${ }^{\mathrm{f}}$, S. Mele ${ }^{\mathrm{q}}$, L. Merola ${ }^{\mathrm{ab}}$, M. Meschini ${ }^{\mathrm{p}}$, W.J. Metzger ad ${ }^{\text {ad }}$ M. von der Mey ${ }^{\text {a }}$, A. Mihul ${ }^{1}$, H. Milcent ${ }^{\mathrm{q}}$, G. Mirabelli ai , J. Mnich ${ }^{\mathrm{q}}$, G.B. Mohanty ${ }^{\mathrm{j}}$, P. Molnar ${ }^{\mathrm{h}}$, B. Monteleoni ${ }^{\mathrm{p}, 3}$, T. Moulik j, G.S. Muanza x , F. Muheim s, A.J.M. Muijs b , M. Musy ai, M. Napolitano ${ }^{\text {ab }}$, F. Nessi-Tedaldi au, H. Newman ${ }^{\text {ae }}$, T. Niessen ${ }^{\text {a }}$, A. Nisati ${ }^{\text {ai }}$, H. Nowak ${ }^{\text {at }}$, Y.D. Oh ${ }^{\text {ao }}$, G. Organtini ${ }^{\text {ai }}$, A. Oulianov ${ }^{\text {aa }}$, C. Palomares ${ }^{y}$, D. Pandoulas a ${ }^{\text {a }}$ S. Paoletti ${ }^{\text {ai, }}$, P. Paolucci ${ }^{\text {ab }}$, R. Paramatti ai, H.K. Park ${ }^{\text {ag }}$, I.H. Park ${ }^{\text {ao }}$, G. Pascale ${ }^{\text {ai }}$, G. Passaleva ${ }^{\text {q }, ~ S . ~ P a t r i c e l l i ~}{ }^{\text {ab }}$, T. Paul ${ }^{\text {k, }}$ M. Pauluzzi af, C. Paus ${ }^{\mathrm{q}}$, F. Pauss ${ }^{\text {au }}$, M. Pedace ${ }^{\text {ai }}$, S. Pensotti ${ }^{\mathrm{z}}$, D. Perret-Gallix ${ }^{d}$, B. Petersen ${ }^{\text {ad }}$, D. Piccolo ${ }^{a b}$, F. Pierella ${ }^{i}$, M. Pieri ${ }^{p}$, P.A. Piroué ${ }^{\text {ah }}$, E. Pistolesi ${ }^{\mathrm{z}}$, V. Plyaskin ${ }^{\text {aa }}$, M. Pohl ${ }^{\mathrm{s}}$, V. Pojidaev ${ }^{\text {aa, }}{ }^{\text {, }}$ H. Postema ${ }^{\mathrm{n}}$, J. Pothier ${ }^{\mathrm{q}}$, N. Produit ${ }^{\text {s, D.O. Prokofiev }}{ }^{\text {ar }}$, D. Prokofiev aj, J. Quartieri ${ }^{\text {ak }}$, G. Rahal-Callot au,q , M.A. Rahaman ${ }^{\mathrm{j}}$, P. Raics ${ }^{\circ}$, N. Raja ${ }^{\mathrm{j}}$, R. Ramelli au', P.G. Rancoita ${ }^{\text {z }}$, A. Raspereza ${ }^{\text {at }}$, G. Raven ${ }^{\text {al }}$, P. Razis ${ }^{\text {ac }}$, D. Ren ${ }^{\text {au }}$, M. Rescigno ${ }^{\text {ai }}$, S. Reucroft ${ }^{\text {k }}$, T. van Rhee ${ }^{\text {aq }}$, S. Riemann ${ }^{\text {at }}$, K. Riles ${ }^{\text {c }}$, A. Robohm ${ }^{\text {au }}$, J. Rodin ${ }^{\text {ap }}$, B.P. Roe ${ }^{\mathrm{c}}$, L. Romero ${ }^{\mathrm{y}}$, A. Rosca ${ }^{\mathrm{h}}$, S. Rosier-Lees ${ }^{\mathrm{d}}$, J.A. Rubio ${ }^{\mathrm{q}}$,

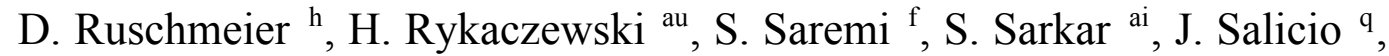

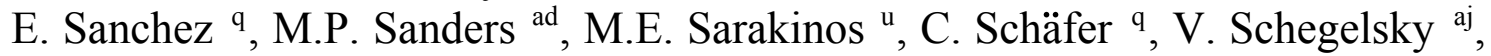
S. Schmidt-Kaerst ${ }^{\text {a }}$, D. Schmitz ${ }^{\text {a }}$, H. Schopper ${ }^{\text {av }}$, D.J. Schotanus ${ }^{\text {ad }}$,


S. Shevchenko ${ }^{\text {ae }}$, N. Shivarov ${ }^{\text {an }}$, V. Shoutko ${ }^{\text {aa }}$, E. Shumilov ${ }^{\text {aa }}$, A. Shvorob ${ }^{\text {ae }}$, T. Siedenburg ${ }^{\text {a }}$, D. Son ${ }^{\text {ao }}$, B. Smith ${ }^{\text {ag }}$, P. Spillantini ${ }^{\text {p }}$, M. Steuer ${ }^{n}$, 
D.P. Stickland ${ }^{\text {ah }}$, A. Stone ${ }^{\text {f }}$, H. Stone ${ }^{\text {ah,3 }}$, B. Stoyanov ${ }^{\text {an }}$, A. Straessner ${ }^{\text {a }}$, K. Sudhakar ${ }^{j}$, G. Sultanov ${ }^{\text {r }}$, L.Z. Sun ${ }^{\text {t }}$, H. Suter ${ }^{\text {au }}$, J.D. Swain ${ }^{\mathrm{r}}$, Z. Szillasi ${ }^{\text {ap, }}$, T. Sztaricskai ${ }^{\text {ap }, 4}$, X.W. Tang ${ }^{\text {g }}$, L. Tauscher ${ }^{\mathrm{e}}$, L. Taylor ${ }^{\mathrm{k}}$, C. Timmermans ${ }^{\text {ad }}$, Samuel C.C. Ting ${ }^{n}$, S.M. Ting ${ }^{n}$, S.C. Tonwar ${ }^{\text {j, J. Tóth }}{ }^{\mathrm{m}}$, C. Tully ${ }^{\mathrm{q}}$, K.L. Tung ${ }^{\mathrm{g}}$, Y. Uchida ${ }^{\mathrm{n}}$, J. Ulbricht ${ }^{\mathrm{au}}$, E. Valente ${ }^{\text {ai }}$, G. Vesztergombi ${ }^{\mathrm{m}}$, I. Vetlitsky ${ }^{\text {aa }}$, D. Vicinanza ${ }^{\text {ak }}$, G. Viertel ${ }^{\text {au }}$, S. Villa ${ }^{\text {k }}$, M. Vivargent ${ }^{d}$, S. Vlachos ${ }^{\text {e }}$, I. Vodopianov aj, H. Vogel ${ }^{\text {ag }}, \mathrm{H}$. Vogt ${ }^{\text {at }}$, I. Vorobiev ${ }^{\text {aa }}$, A.A. Vorobyov ${ }^{\text {aj, }}$, A. Vorvolakos ${ }^{\text {ac }}$, M. Wadhwa ${ }^{\mathrm{e}}$, W. Wallraff ${ }^{\mathrm{a}}$, M. Wang ${ }^{\mathrm{n}}$, X.L. Wang ${ }^{\mathrm{t}}$, Z.M. Wang ${ }^{\mathrm{t}}$, A. Weber ${ }^{\mathrm{a}}$, M. Weber ${ }^{\mathrm{a}}$, P. Wienemann ${ }^{\mathrm{a}}$, H. Wilkens ${ }^{\text {ad }}$, S.X. Wu ${ }^{\mathrm{n}}$, S. Wynhoff ${ }^{\mathrm{q}}$, L. Xia ${ }^{\text {ae }}$, Z.Z. Xu ${ }^{\mathrm{t}}$, B.Z. Yang ${ }^{\mathrm{t}}$, C.G. Yang ${ }^{\mathrm{g}}$, H.J. Yang ${ }^{\mathrm{g}}$, M. Yang ${ }^{\text {g }}$, J.B. Ye ${ }^{\mathrm{t}}$, S.C. Yeh ${ }^{\text {ax }}$, An. Zalite ${ }^{\text {aj }}$, Yu. Zalite ${ }^{\text {aj }}$, Z.P. Zhang ${ }^{\mathrm{t}}$, G.Y. Zhu ${ }^{\text {g }}$, R.Y. Zhu ${ }^{\text {ae }}$, A. Zichichi ${ }^{\mathrm{i}, \mathrm{q}, \mathrm{r}}$, G. Zilizi ${ }^{\text {ap, }}{ }^{4}$, M. Zöller ${ }^{\mathrm{a}}$

${ }^{a}$ I. Physikalisches Institut, RWTH, D-52056 Aachen, Germany, and III. Physikalisches Institut, RWTH, D-52056 Aachen, Germany 5

${ }^{\mathrm{b}}$ National Institute for High Energy Physics, NIKHEF, and University of Amsterdam, NL-1009 DB Amsterdam, The Netherlands ${ }^{c}$ University of Michigan, Ann Arbor, MI 48109, USA

d Laboratoire d'Annecy-le-Vieux de Physique des Particules, LAPP, IN2P3-CNRS, BP 110, F-74941 Annecy-le-Vieux CEDEX, France

${ }^{\mathrm{e}}$ Institute of Physics, University of Basel, CH-4056 Basel, Switzerland

${ }^{\mathrm{f}}$ Louisiana State University, Baton Rouge, LA 70803, USA

${ }^{\mathrm{g}}$ Institute of High Energy Physics, IHEP, 100039 Beijing, China ${ }^{6}$

${ }^{\mathrm{h}}$ Humboldt University, D-10099 Berlin, Germany ${ }^{5}$

${ }^{\mathrm{i}}$ University of Bologna and INFN-Sezione di Bologna, I-40126 Bologna, Italy

${ }^{\mathrm{j}}$ Tata Institute of Fundamental Research, Bombay 400 005, India

${ }^{\mathrm{k}}$ Northeastern University, Boston, MA 02115, USA

${ }^{1}$ Institute of Atomic Physics and University of Bucharest, R-76900 Bucharest, Romania

${ }^{\mathrm{m}}$ Central Research Institute for Physics of the Hungarian Academy of Sciences, H-1525 Budapest 114, Hungary ${ }^{7}$

${ }^{\mathrm{n}}$ Massachusetts Institute of Technology, Cambridge, MA 02139, USA

${ }^{\circ}$ KLTE-ATOMKI, H-4010 Debrecen, Hungary ${ }^{4}$

${ }^{\mathrm{p}}$ INFN Sezione di Firenze and University of Florence, I-50125 Florence, Italy

${ }^{\mathrm{q}}$ European Laboratory for Particle Physics, CERN, CH-1211 Geneva 23, Switzerland

${ }^{\mathrm{r}}$ World Laboratory, FBLJA Project, CH-1211 Geneva 23, Switzerland

${ }^{\mathrm{s}}$ University of Geneva, $\mathrm{CH}-1211$ Geneva 4, Switzerland

${ }^{\mathrm{t}}$ Chinese University of Science and Technology, USTC, Hefei, Anhui 230 029, China ${ }^{6}$

" SEFT, Research Institute for High Energy Physics, P.O. Box 9, SF-00014 Helsinki, Finland

${ }^{v}$ University of Lausanne, CH-1015 Lausanne, Switzerland

${ }^{\mathrm{w}}$ INFN-Sezione di Lecce and Universitá Degli Studi di Lecce, I-73100 Lecce, Italy

${ }^{\mathrm{x}}$ Institut de Physique Nucléaire de Lyon, IN2P3-CNRS, Université Claude Bernard, F-69622 Villeurbanne, France

${ }^{y}$ Centro de Investigaciones Energéticas, Medioambientales y Tecnologícas, CIEMAT, E-28040 Madrid, Spain ${ }^{8}$

${ }^{\mathrm{z}}$ INFN-Sezione di Milano, I-20133 Milan, Italy

${ }^{\text {aa }}$ Institute of Theoretical and Experimental Physics, ITEP, Moscow, Russia

${ }^{\mathrm{ab}}$ INFN-Sezione di Napoli and University of Naples, I-80125 Naples, Italy

ac Department of Natural Sciences, University of Cyprus, Nicosia, Cyprus

${ }^{\text {ad }}$ University of Nijmegen and NIKHEF, NL-6525 ED Nijmegen, The Netherlands

${ }^{\text {ae } C a l i f o r n i a ~ I n s t i t u t e ~ o f ~ T e c h n o l o g y, ~ P a s a d e n a, ~ C A ~ 91125, ~ U S A ~}$

af INFN-Sezione di Perugia and Universitá Degli Studi di Perugia, I-06100 Perugia, Italy

${ }^{\text {ag }}$ Carnegie Mellon University, Pittsburgh, PA 15213, USA

ah Princeton University, Princeton, NJ 08544, USA

ai INFN-Sezione di Roma and University of Rome, "La Sapienza", I-00185 Rome, Italy

aj Nuclear Physics Institute, St. Petersburg, Russia

${ }^{\mathrm{ak}}$ University and INFN, Salerno, I-84100 Salerno, Italy

${ }^{\text {al }}$ University of California, San Diego, CA 92093, USA

${ }^{\text {am }}$ Dept. de Fisica de Particulas Elementales, Univ. de Santiago, E-15706 Santiago de Compostela, Spain 
an Bulgarian Academy of Sciences, Central Lab. of Mechatronics and Instrumentation, BU-1113 Sofia, Bulgaria

${ }^{\text {ao } C e n t e r ~ f o r ~ H i g h ~ E n e r g y ~ P h y s i c s, ~ A d v . ~ I n s t . ~ o f ~ S c i e n c e s ~ a n d ~ T e c h n o l o g y, ~ 305-701 ~ T a e j o n, ~ S o u t h ~ K o r e a ~}$

${ }^{a p}$ University of Alabama, Tuscaloosa, AL 35486, USA

${ }^{\text {aq }}$ Utrecht University and NIKHEF, NL-3584 CB Utrecht, The Netherlands

${ }^{\text {ar }}$ Purdue University, West Lafayette, IN 47907, USA

as Paul Scherrer Institut, PSI, CH-5232 Villigen, Switzerland

${ }^{\text {at }}$ DESY, D-15738 Zeuthen, Germany

au Eidgenössische Technische Hochschule, ETH Zürich, CH-8093 Zürich, Switzerland

av University of Hamburg, D-22761 Hamburg, Germany

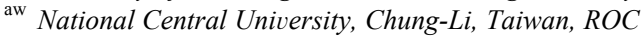

ax Department of Physics, National Tsing Hua University, Taiwan, ROC

Received 16 December 1999; accepted 19 January 2000

Editor: K. Winter

\begin{abstract}
The total and differential cross sections of the process $\mathrm{e}^{+} \mathrm{e}^{-} \rightarrow n \gamma$ with $n \geq 2$ are measured using data collected by the L3 experiment at centre-of-mass energies of $\sqrt{s}=183$ and $189 \mathrm{GeV}$. The results are in agreement with the Standard Model expectations. Limits are set on deviations from QED, contact interaction cut-off parameters and masses of excited electrons. (C) 2000 Published by Elsevier Science B.V. All rights reserved.
\end{abstract}

\section{Introduction}

The process $\mathrm{e}^{+} \mathrm{e}^{-} \rightarrow \gamma \gamma(\gamma)$, where $(\gamma)$ denotes possible additional photons, is described very accurately by QED. The experimental signature of these events is clean, and they can be selected with negligible background. Therefore, this process is well suited to test QED and to look for new physics phenomena, whose expected contributions grow with the increase of the centre-of-mass energy, $\sqrt{s}$.

In this paper, the results on the study of the process $\mathrm{e}^{+} \mathrm{e}^{-} \rightarrow n \gamma(n \geq 2)$ are presented. The anal-

\footnotetext{
${ }^{1}$ Also supported by CONICET and Universidad Nacional de La Plata, CC 67, 1900 La Plata, Argentina.

${ }^{2}$ Also supported by Panjab University, Chandigarh-160014, India.

${ }^{3}$ Deceased.

${ }^{4}$ Also supported by the Hungarian OTKA fund under contract numbers T22238 and T026178.

${ }^{5}$ Supported by the German Bundesministerium für Bildung, Wissenschaft, Forschung und Technologie.

${ }^{6}$ Supported by the National Natural Science Foundation of China.

${ }^{7}$ Supported by the Hungarian OTKA fund under contract numbers T019181, F023259 and T024011.

${ }^{8}$ Supported also by the Comisión Interministerial de Ciencia y Tecnología.
}

ysis is performed on the data sample collected by the L3 detector [1] during 1997 and 1998, at $\sqrt{s}=$ $182.7 \mathrm{GeV}(183 \mathrm{GeV}$ hereafter) and $\sqrt{s}=188.7 \mathrm{GeV}$ ( $189 \mathrm{GeV}$ hereafter) respectively. The integrated luminosities for each sample are $54.8 \mathrm{pb}^{-1}$ and 175.3 $\mathrm{pb}^{-1}$, respectively. Previous results have been published by L3 at lower centre-of-mass energies [2-4] and by other experiments [5].

\section{Event selection}

The analysis performed on these data is similar to that reported in previous papers [4]. A photon candidate is defined as:

- A shower in the electromagnetic barrel or end-cap calorimeters with energy larger than $1 \mathrm{GeV}$. The profile of the shower must be consistent with that of an electromagnetic particle.

- The number of hits in the vertex chamber within an azimuthal angle of $\pm 8^{\circ}$ around the direction of the photon candidate must be less than $40 \%$ of the expected number of hits for a charged particle.

To select an event there must be at least two photon candidates with polar angles $\theta_{\gamma}$ between $16^{\circ}$ 
Table 1

Number of observed, $N_{\text {obs }}$, and expected, $N_{\text {exp }}$, events with 2, 3 and 4 photons

\begin{tabular}{|c|c|c|c|c|}
\hline & \multicolumn{2}{|c|}{$\sqrt{s}=183 \mathrm{GeV}$} & \multicolumn{2}{|c|}{$\sqrt{s}=189 \mathrm{GeV}$} \\
\hline & $N_{\mathrm{obs}}$ & $\overline{N_{\exp }}$ & $N_{\text {obs }}$ & $N_{\text {exp }}$ \\
\hline$\overline{2 \gamma}$ & 436 & 453 & 1302 & 1346 \\
\hline $3 \gamma$ & 23 & 24 & 72 & 69 \\
\hline $4 \gamma$ & 1 & 0.04 & 0 & 0.1 \\
\hline
\end{tabular}

and $164^{\circ}$ with an angular separation of more than $15^{\circ}$ and no other activity in the detector. In addition, to reject $\mathrm{e}^{+} \mathrm{e}^{-} \rightarrow \nu \bar{\nu} \gamma \gamma$ and cosmic rays, we require that the sum of the energies of the photon candidates be larger than $\sqrt{s} / 2$.

The only expected backgrounds are $\mathrm{e}^{+} \mathrm{e}^{-} \rightarrow$ $\mathrm{e}^{+} \mathrm{e}^{-}(\gamma)$ and $\mathrm{e}^{+} \mathrm{e}^{-} \rightarrow \tau^{+} \tau^{-}(\gamma)$. These contributions are estimated from Monte Carlo simulations using BHWIDE [6] for Bhabha events and KORALZ [7] for $\tau$ events, and are found to be negligible. The acceptance is computed applying the same analysis to a sample of $\mathrm{e}^{+} \mathrm{e}^{-} \rightarrow \gamma \gamma(\gamma)$ events generated using an order $\alpha^{3}$ Monte Carlo generator [8] passed through the L3 simulation [9] and reconstruction programs. The selection efficiencies to detect at least two photons inside the fiducial volume are found to

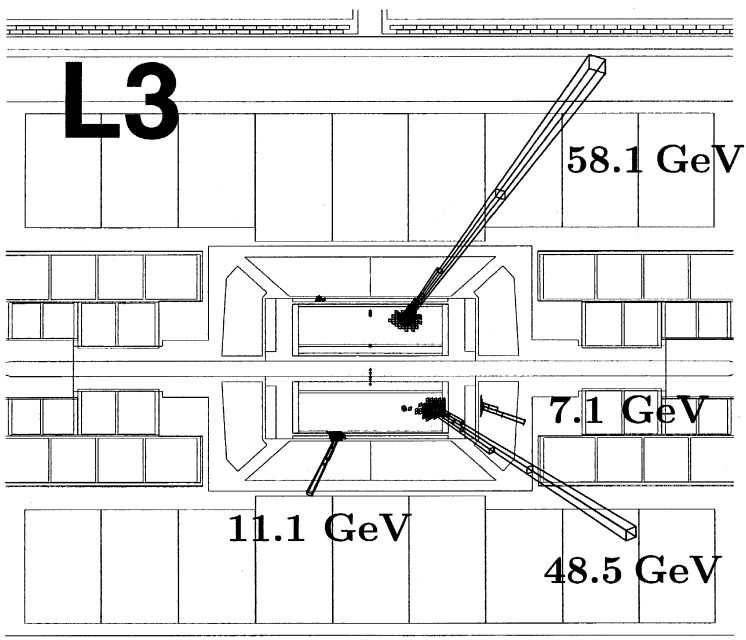

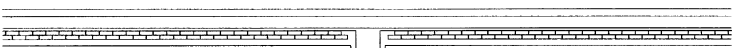

Fig. 1. Display of an event with four detected photons at $\sqrt{s}=$ $183 \mathrm{GeV}$. be $68.8 \pm 0.2 \%$ at $\sqrt{s}=183 \mathrm{GeV}$ and $68.0 \pm 0.2 \%$ at $\sqrt{s}=189 \mathrm{GeV}$ for $16^{\circ}<\theta_{\gamma}<164^{\circ}$, where the errors quoted are the statistical errors of the Monte Carlo sample. The efficiency of the calorimetric energy trigger is estimated to be above $99.7 \%$ for both samples. It is estimated by using a sample of Bhabha events, which has an independent trigger for charged particles.

\section{Analysis}

A total of 460 events at $\sqrt{s}=183 \mathrm{GeV}$ and 1374 events at $\sqrt{s}=189 \mathrm{GeV}$ are selected. They are classified according to the number of isolated photons in $16^{\circ}<\theta_{\gamma}<164^{\circ}$, as presented in Table 1 , together with the number of expected events. Fig. 1 shows
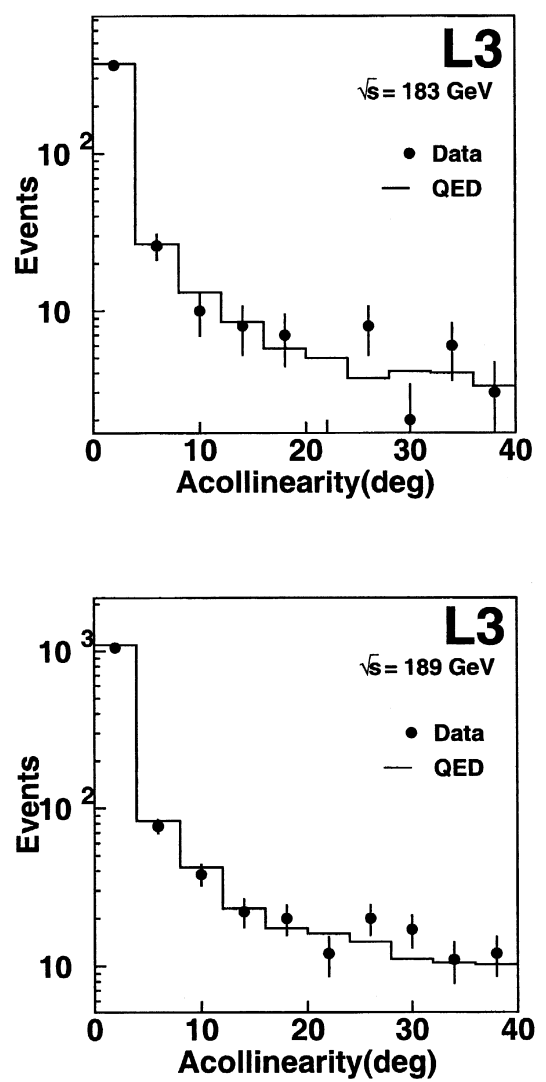

Fig. 2. Distribution of the acollinearity angle between the two most energetic photons in the $\mathrm{e}^{+} \mathrm{e}^{-} \rightarrow \gamma \gamma(\gamma)$ process at $\sqrt{s}=$ $183 \mathrm{GeV}$ (top), and $\sqrt{s}=189 \mathrm{GeV}$ (bottom). 
one event with 4 detected photons at $\sqrt{s}=183 \mathrm{GeV}$. No events with 5 or more photons in this angular range have been observed. For the two most energetic photons, the acollinearity angle distribution is shown in Fig. 2, and the acoplanarity angle distribution in Fig. 3.

The differential cross section as a function of the $\cos \theta$ of the event is shown in Fig. 4. The polar angle $\theta$ of the event is defined as

$\cos \theta=\left|\sin \left(\frac{\theta_{1}-\theta_{2}}{2}\right) / \sin \left(\frac{\theta_{1}+\theta_{2}}{2}\right)\right|$,

where $\theta_{1}$ and $\theta_{2}$ are the polar angles of the two most energetic photons in the event. The measured differential distributions have been corrected for efficiency and higher order QED contributions using the Monte Carlo simulation. These distributions are then compared directly with the lowest order QED predic-


Fig. 3. Distribution of the acoplanarity angle between the two most energetic photons in the $\mathrm{e}^{+} \mathrm{e}^{-} \rightarrow \gamma \gamma(\gamma)$ process at $\sqrt{s}=$ $183 \mathrm{GeV}$ (top), and $\sqrt{s}=189 \mathrm{GeV}$ (bottom).
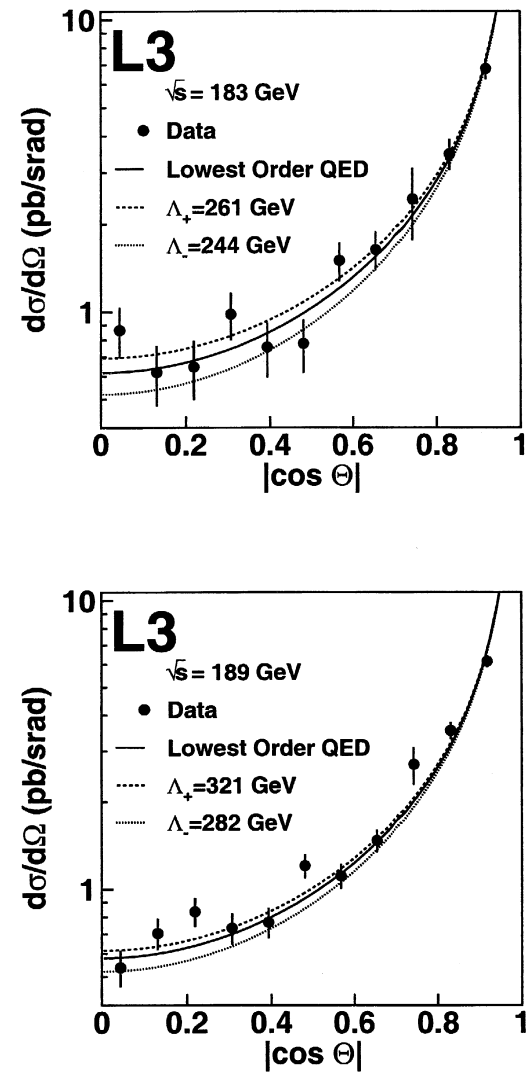

Fig. 4. Differential cross section as a function of $\cos \theta$ for the process $\mathrm{e}^{+} \mathrm{e}^{-} \rightarrow \gamma \gamma(\gamma)$. The points show the measurements corrected for efficiency and additional photons. The solid line corresponds to the lowest order QED prediction. The dashed and dotted lines represent the limits obtained for deviations from QED, taking into account all the L3 data at centre-of-mass energies up to that presented in the corresponding plot.

tions. Good agreement between the data and the QED prediction is observed.

The observed number of events corresponds to a total cross section in the fiducial region $16^{\circ}<\theta<$ $164^{\circ}$ of:

$\sigma_{\gamma \gamma(\gamma)}=12.17 \pm 0.55 \pm 0.14 \mathrm{pb} \quad(\sqrt{s}=183 \mathrm{GeV})$,

$\sigma_{\gamma \gamma(\gamma)}=11.54 \pm 0.30 \pm 0.14 \mathrm{pb} \quad(\sqrt{s}=189 \mathrm{GeV})$,

where the first error is statistical and the second is systematic. The main source of systematic error is the uncertainty in the selection efficiency. It has been evaluated by varying the selection cuts and taking into account the finite Monte Carlo statistics. The systematic error coming from the uncertainty in the 


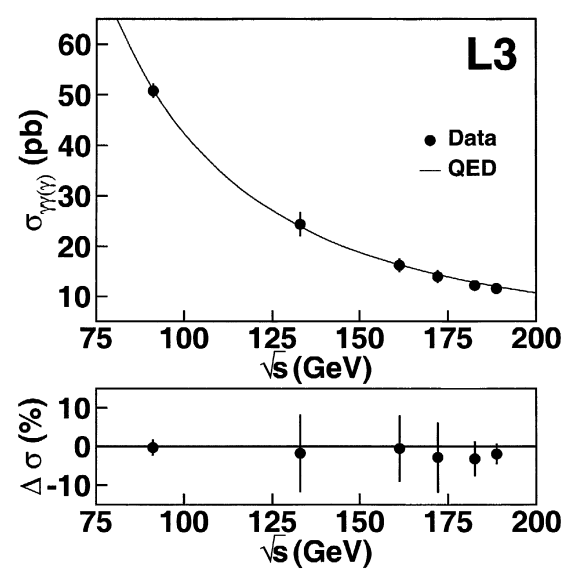

Fig. 5. Measured cross section as a function of the centre-of-mass energy for $\theta$ between $16^{\circ}$ and $164^{\circ}$ compared with the QED prediction. The value at $\sqrt{s}=91 \mathrm{GeV}$ has been extrapolated to this angular range from the one given in [2]. The bottom part of the figure presents the relative deviation of the measurements with respect to the QED expectations.

measured luminosity $( \pm 0.2 \%)$ and in the background present in the sample $(<0.5 \%)$ are found to be negligible. The statistical error dominates in the measurement of the cross section both at $183 \mathrm{GeV}$ and at $189 \mathrm{GeV}$. The QED predicted cross sections are $12.65 \mathrm{pb}$ and $11.78 \mathrm{pb}$ [8] respectively, in agreement with the measurements.

These cross sections and previously measured values [2-4] together with the QED prediction, are presented in Fig. 5 as a function of the centre-of-mass energy.

\section{Limits on deviations from QED}

The possible deviations from QED are parametrised by effective Lagrangians, and their effect on the observables can be expressed as a multiplicative correction term to the QED differential cross section. Depending on the type of Lagrangian, two general forms are considered [10]:

$\frac{d \sigma}{d \Omega}=\left(\frac{d \sigma}{d \Omega}\right)_{\mathrm{QED}}\left(1+\frac{s^{2}}{\alpha} \frac{1}{\Lambda^{4}} \sin ^{2} \theta\right)$,

and

$$
\frac{d \sigma}{d \Omega}=\left(\frac{d \sigma}{d \Omega}\right)_{\mathrm{QED}}\left(1+\frac{s^{3}}{32 \pi \alpha^{2}} \frac{1}{\Lambda^{\prime}} \frac{\sin ^{2} \theta}{1+\cos ^{2} \theta}\right) .
$$

The correction factors depend on the centre-of-mass energy, the polar angle and the scale parameters $\Lambda$, $\Lambda^{\prime}$ which have dimensions of energy. A more standard way of parametrising the deviations from QED is the introduction of the cut-off parameters $\Lambda_{ \pm}$ [11]. The differential cross section can be obtained from Eq. (1) by replacing $\Lambda^{4}$ by $\pm(2 / \alpha) \Lambda_{+}^{4}$.

Limits on the different scale parameters have already been set in our previous publications [3,4]. However, since the sensitivity to possible deviations from QED increases rapidly with the centre-of-mass energy they are superseded by the present data. In order to quantify the possible deviations from QED we perform a maximum likelihood fit to the differential cross sections at each centre-of-mass energy. The estimated parameters combining the present results with those in our previous analyses [3,4] are:

$$
\begin{aligned}
& \frac{1}{\Lambda^{4}}=\left(-0.019_{-0.038}^{+0.054}\right) \times 10^{-11} \mathrm{GeV}^{-4}, \\
& \frac{1}{\Lambda^{\prime}}=\left(-0.048_{-0.092}^{+0.131}\right) \times 10^{-16} \mathrm{GeV}^{-6},
\end{aligned}
$$

consistent with no deviations from QED. To determine the confidence levels, the probability distribution is normalised over the physically allowed range of the parameters. At the $95 \%$ C.L. the following limits are obtained:

$\Lambda>1304 \mathrm{GeV}$, $\Lambda_{+}>321 \mathrm{GeV}$,

$\Lambda^{\prime}>703 \mathrm{GeV}$, $\Lambda_{-}>282 \mathrm{GeV}$.

The effects of $\Lambda_{ \pm}$in the differential cross section can be seen in Fig. 4 . In this case, the parameters $\Lambda_{ \pm}$have been fixed to the limits values quoted before.

The existence of excited electrons $\left(\mathrm{e}^{*}\right)$ would also introduce deviations from the QED predictions in the $\gamma \gamma(\gamma)$ final states. The excited electron, of mass $m_{\mathrm{e}^{*}}$, couples to e and $\gamma$ via two possible interactions. The first is purely magnetic [12],

$\mathscr{L}=\frac{e}{2 \Lambda_{\mathrm{e}^{*}}} \bar{\Psi}_{\mathrm{e}^{*}} \sigma^{\mu \nu} \Psi_{\mathrm{e}} F_{\mu \nu}+$ h.c.

and the second is a chiral-magnetic one [13]:

$\mathscr{L}=\frac{e}{2 \Lambda_{\mathrm{e}^{*}}} \bar{\Psi}_{\mathrm{e}^{*}} \sigma^{\mu \nu}\left(1 \pm \gamma^{5}\right) \Psi_{\mathrm{e}} F_{\mu \nu}+$ h.c. 
In both cases we fit the excited electron mass fixing the interaction scale $\Lambda_{\mathrm{e}^{*}}$ to $m_{\mathrm{e}^{*}}$, obtaining

Purely Magnetic: $\frac{1}{m_{\mathrm{e}^{*}}^{4}}=\left(-0.052_{-0.104}^{+0.143}\right)$

$$
\times 10^{-9} \mathrm{GeV}^{-4},
$$

Chiral-Magnetic: $\quad \frac{1}{m_{\mathrm{e}^{*}}^{4}}=\left(-0.135_{-0.352}^{+0.383}\right)$

$$
\times 10^{-9} \mathrm{GeV}^{-4} \text {. }
$$

From them we derive the $95 \%$ C.L. lower limits of:

Purely Magnetic: $m_{\mathrm{e}^{*}}>283 \mathrm{GeV}$,

Chiral-Magnetic: $\quad m_{\mathrm{e}^{*}}>213 \mathrm{GeV}$.

\section{Acknowledgements}

We wish to express our gratitude to the CERN accelerator divisions for the excellent performance of the LEP machine. We acknowledge the effort of the engineers and technicians who have participated in the construction and maintenance of the experiment.

\section{References}

[1] L3 Collaboration, B. Adeva et al., Nucl. Instr. and Meth. A 289 (1990) 35; M. Chemarin et al., Nucl. Instr. and Meth. A
349 (1994) 345; M. Acciarri et al., Nucl. Instr. and Meth. A 351 (1994) 300; G. Basti et al., Nucl. Instr. and Meth. A 374 (1996) 293; I.C. Brock et al., Nucl. Instr. and Meth. A 381 (1996) 236; A. Adam et al., Nucl. Instr. and Meth. A 383 (1996) 342.

[2] L3 Collaboration, M. Acciarri et al., Phys. Lett. B 353 (1995) 136.

[3] L3 Collaboration, M. Acciarri et al., Phys. Lett. B 384 (1996) 323.

[4] L3 Collaboration, M. Acciarri et al., Phys. Lett. B 413 (1997) 159.

[5] ALEPH Collaboration, D. Buskulic et al., Phys. Lett. B 429 (1998) 201. DELPHI Collaboration, P. Abreu et al., Phys. Lett. B 433 (1998) 429. OPAL Collaboration, K. Ackerstaff et al., Phys. Lett. B 465 (1999) 303.

[6] S. Jadach et al., Phys. Lett. B 390 (1997) 298.

[7] S. Jadach et al., Comp. Phys. Comm. 79 (1994) 503.

[8] F.A. Berends, R. Kleiss, Nucl. Phys. B 186 (1981) 22; CALKUL Collaboration, F.A. Berends et al., Nucl. Phys. B 239 (1984) 395.

[9] The L3 detector simulation is based on GEANT Version 3.15, R. Brun et al., CERN DD/EE/84-1 (Revised 1987); The GHEISHA program (H. Fesefeldt, RWTH Aachen Report PITHA $85 / 02(1985))$ is used to simulate hadronic interactions.

[10] O.J.P. Eboli et al., Phys. Lett. B 271 (1991) 274.

[11] F.E. Low, Phys. Rev. Lett. 14 (1965) 238; R.P. Feynman, Phys. Rev. Lett. 74 (1948) 939; F.M. Renard, Phys. Lett. B 116 (1982) 264; S. Drell, Ann. Phys. (NY) 4 (1958) 75.

[12] A. Litke, Harvard Univ., Ph.D. thesis, 1970, unpublished.

[13] K. Hagiwara, S. Komamyia, D. Zeppenfeld, Z. Phys. C 29 (1985) 115; N. Cabibbo, L. Maiani, Y. Srivastava, Phys. Lett B 139 (1984) 459; F.M. Renard, Nucl. Phys. B 196 (1982) 93. 